\title{
A NEW LOOK AT THE 2D ISING MODEL FROM EXACT PARTITION FUNCTION ZEROS FOR LARGE LATTICE SIZES
}

\author{
NELSON A. ALVES* and J. R. DRUGOWICH DE FELICIO ${ }^{\dagger}$ \\ Departamento de Física e Matemática, FFCLRP, Universidade de São Paulo \\ Av. Bandeirantes 3900, CEP 014040-901 Ribeirão Preto, SP, Brazil \\ ULRICH H. E. HANSMANN $\ddagger$ \\ Department of Theoretical Studies, Institute for Molecular Science \\ Okazaki, Aichi 444, Japan
}

\begin{abstract}
A general numerical method is presented to locate the partition function zeros in the complex $\beta$ plane for large lattice sizes. We apply this method to the $2 \mathrm{D}$ Ising model and results are reported for square lattice sizes up to $L=64$. We also propose an alternative method to evaluate corrections to scaling which relies only on the leading zeros. This method is illustrated with our data.
\end{abstract}

\section{Introduction}

Although the two-dimensional Ising model is exactly solved for zero external field, it continues receiving attention in many aspects. In a recent work by Beale ${ }^{1}$ for instance, the low-temperature series expansion for the partition function was exactly determined for finite lattices with periodic boundary conditions. In terms of the expansion variable $u=e^{-4 \beta}$ the partition function on a $m \times n$ lattice size becomes a polynomial of finite degree in $u$, and its coefficients $g(E)$, the number of configurations with energy $E$, were calculated from an exact closed form based on Kaufman's solution. ${ }^{2}$

Motivated by that calculation as well by the enhancement of computer facilities we decided to revisit the $2 \mathrm{D}$ Ising model to obtain the exact partition function zeros in the complex temperature plane. ${ }^{3}$ This approach had already been pursued by Katsura and $\mathrm{Abe}^{4,5}$ in the early investigations of zero distributions in order to check the proposal by Fisher about their loci. ${ }^{6}$ Other papers have aimed the study of the critical properties from the leading zeros, but all of them were limited to small lattices $(m \times n \leq 13 \times 13) .^{7,8,9}$

In this work we present a procedure to obtain exact complex zeros of the partition function for large lattice sizes. We provide a description of a way round technical limitations on solving polynomials, at least in what concerns the location of the first zeros $\left(u_{1}^{0}, u_{2}^{0}, \ldots\right)$. It is a modified version of the scanning procedure which has been applied to continuous energy distributions of lattice gauge theories. ${ }^{10,11,12}$

\footnotetext{
*E-mail: alves@fma.if.usp.br

${ }^{\dagger}$ E-mail: drugo@pinguim.ffclrp.usp.br

${ }^{\ddagger}$ E-mail: hansmann@ims.ac.jp
} 
Our approach is based on constructing a new function in terms of $\ln g(E)$. It is presented in section 2 , where the first exact zeros are calculated for square lattice sizes up to $L=64$. Since we are working with numerical computation, we mean by exact zeros accurate values limited only by the use of double-precision floating-point arithmetic.

The partition function zeros approach has been largely used to obtain information on phase transitions from Monte Carlo (MC) simulations ${ }^{3,10,13}$ or exact enumerations ${ }^{9}$ of finite systems. In this context, Itzykson's et al. ${ }^{3}$ finite size scaling (FSS) relation for the first zero is the proper way to calculate the correlation exponent $\nu$. We present results so obtained in section 3 . In addition, looking forward to obtain more information from those exact zeros, we propose, in the same section, a new way to evaluate corrections to FSS which relies only on $u_{1}^{0}(L)$ data.

\section{Exact partition function zeros}

The partition function of the two-dimensional Ising model on a $m \times n$ lattice can be written as a polynomial,

$$
Z_{n, m}(\beta)=e^{2 n m \beta} \sum_{E=0}^{n m} g(E) u^{E}
$$

where $u=e^{-4 \beta}$.

Kaufman's solution for the isotropic Ising model renders the analytical expressions to be expressed in the polynomial form (11). Following Beale ${ }^{1}$, this can be done for any lattice size by using MATHEMATICA.

A further step namely exact determination of their zeros and FSS analysis for the leading ones, can be achieved with this polynomial form. However we have checked that it was not possible to handle systems for $L$ larger than 16 with our workstations. In fact, as the lattice size increases, the exact coefficients become very large integers. The enormous increasing of their maximum values, typically $\ln g(E) \simeq 174$ for $L=16$ and going up to $\ln g(E) \simeq 2835$ for $L=64$, prevent us from solving them by using computer algebra language. The same reasons do not indicate the use of standard numerical algorithms, ${ }^{14,15}$ usually employed in those cases.

To circumvent this problem we borrowed inspiration from lattice gauge theories where the energy distributions are continuous. There, in contrast to spin systems where the action takes discrete values and the partition function becomes a polynomial in $u$, a time series analysis in function of the complex coupling $\beta=\beta_{x}+i \beta_{y}$ is more efficient in calculating the first zero which is closest to the infinite volume critical point. It is a two step approach. ${ }^{12}$ First, we scan graphically for separate zeros of $\operatorname{Re} Z(\beta)$ and $\operatorname{Im} Z(\beta)$, where

$$
Z(\beta)=\sum_{E} g(E) u^{\triangle E}
$$


The shift $\triangle E=E-\langle E\rangle$ is usually introduced for technical reasons, to avoid numerical overflow computations, although it is not any more relevant in the new approach. A typical output is shown in Fig. 1. Crosses correspond to $\operatorname{Re} Z(\beta)=0$ and diamonds to $\operatorname{Im} Z(\beta)=0$. The wanted zero is obtained when the lines cross. Second, we compute this zero to a desired precision as an iterative process. This can be achieved by means of the minimization algorithm AMOEBA ${ }^{16}$ for the function $\left((\operatorname{Re} Z(\beta))^{2}+(\operatorname{Im} Z(\beta))^{2}\right)^{1 / 2}$, whose starting point is obtained from a simple inspection of figures like Fig. 1. As an example, we can use the input $(0.43765,0.0131)$ as the starting point to this routine which leads, after roughly 100 iterations, to $\left(\beta_{x}^{0}, \beta_{y}^{0}\right)=(0.4376431265,0.01311604331)$.

Now we shall describe how to implement our approach.

Since our aim is to achieve large lattices one has to work with logarithms. For this end we need to introduce a new function $F(\beta)=F_{x}(\beta)+i F_{y}(\beta)$ to play the role of $Z(\beta)$ itself. We start from splitting $\operatorname{Re} Z(\beta)$ into two positive parts, namely

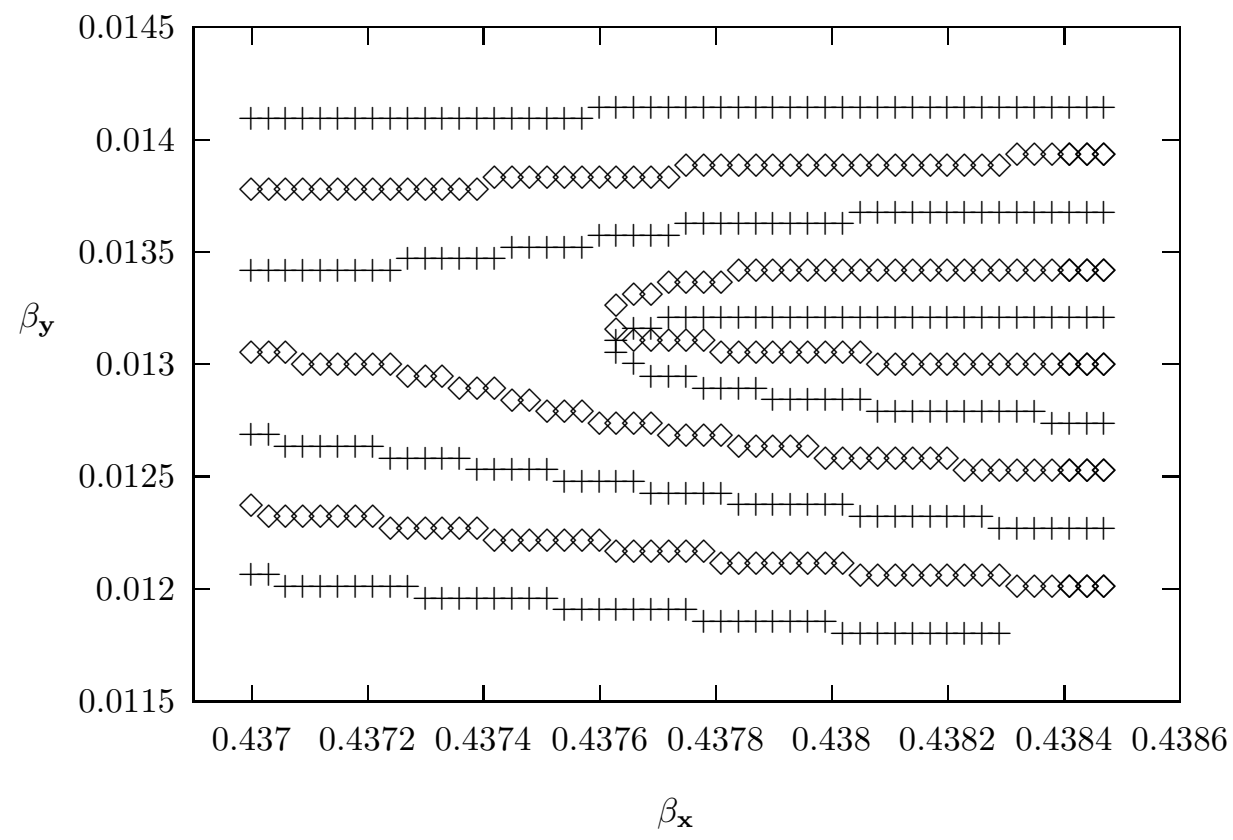

Fig. 1. Search for the first partition function zero for $L=64$. The crosses indicate the zeros for $\operatorname{Re} F(\beta)$ and the diamonds the ones for $\operatorname{Im} F(\beta)$. The complex function $F(\beta)$ has the same zeros as $Z(\beta)$.

$G_{E}$ and $H_{E}$, defined by

$$
\operatorname{Re} Z(\beta)=G_{E}-H_{E}
$$


A New Look at the 2D Ising Model

where

$$
\begin{gathered}
G_{E}=\sum_{E}^{\prime} g(E) e^{-4 \beta_{x} \triangle E} \cos \left(\alpha_{E}\right), \\
H_{E}=\sum_{E}^{\prime \prime} g(E) e^{-4 \beta_{x} \Delta E}\left|\cos \left(\alpha_{E}\right)\right|,
\end{gathered}
$$

and $\alpha_{E}=4 \beta_{y} \triangle E$. Here $\sum^{\prime}$ means a summation over $E$ provided $\cos \left(\alpha_{E}\right)>0$, and $\sum^{\prime \prime}$ stands for the complementary values where $\cos \left(\alpha_{E}\right)<0$. Next we calculate $\ln G_{E}$ and $\ln H_{E}$ in a recursive way from the terms $\ln g(E), \ln \cos \left(\alpha_{E}\right)$ and $-4 \beta_{x} \triangle E$. Logarithmic terms can be added up two by two in $G_{E}$ and $H_{E}$, respectively, by using the relation

$$
\ln (a+b)=\ln b+\ln \left(1+e^{\ln a-\ln b}\right) .
$$

Finally, since we are interested in the $\beta$ values where $\operatorname{Re} Z(\beta)$ and $\operatorname{Im} Z(\beta)$ change signals, we realize that this can be achieved by the function

$$
F_{x}(\beta)=\ln G_{E}-\ln H_{E}
$$

and a similar one for $F_{y}(\beta)$, which follows from the imaginary part of $Z(\beta)$.

Now, we apply the two steps procedure to find roots of $F_{x}(\beta)$ and $F_{y}(\beta)$ instead of $\operatorname{Re} Z(\beta)$ and $\operatorname{Im} Z(\beta)$. In Fig. 1 we show the first step for $L=64$. Table 1 contains our leading zeros $u_{1}^{0}(L)$ for lattice sizes up to $L=64$, with rounded errors in the last digit.

Table 1. First partition function zeros.

\begin{tabular}{ccc}
\hline$L$ & $\operatorname{Re}\left(u_{1}^{0}\right)$ & $\operatorname{Im}\left(u_{1}^{0}\right)$ \\
\hline 4 & 0.1624473772 & 0.16648190032 \\
6 & 0.1756913616 & 0.10528348725 \\
8 & 0.1780809275 & 0.07710375572 \\
9 & 0.1783370200 & 0.06801661701 \\
10 & 0.1783571854 & 0.06084948478 \\
12 & 0.1780873239 & 0.05026266796 \\
15 & 0.1774653671 & 0.03986421638 \\
16 & 0.1772557409 & 0.03729300267 \\
18 & 0.1768587209 & 0.03303236187 \\
20 & 0.1764984476 & 0.02964570468 \\
24 & 0.1758873488 & 0.02460155919 \\
30 & 0.1751918649 & 0.01959967784 \\
32 & 0.1750048586 & 0.01835571819 \\
36 & 0.1746815338 & 0.01628818111 \\
40 & 0.1744124041 & 0.01463927731 \\
48 & 0.1739912845 & 0.01217440355 \\
60 & 0.1735492820 & 0.00971963267 \\
64 & 0.1734355215 & 0.00910750889 \\
$\infty$ & 0.1715728753 & \\
\hline
\end{tabular}

This method can easily be implemented and takes few minutes of CPU time for a Fortran code in a workstation after we have calculated the coefficients $g(E) .^{1}$ 


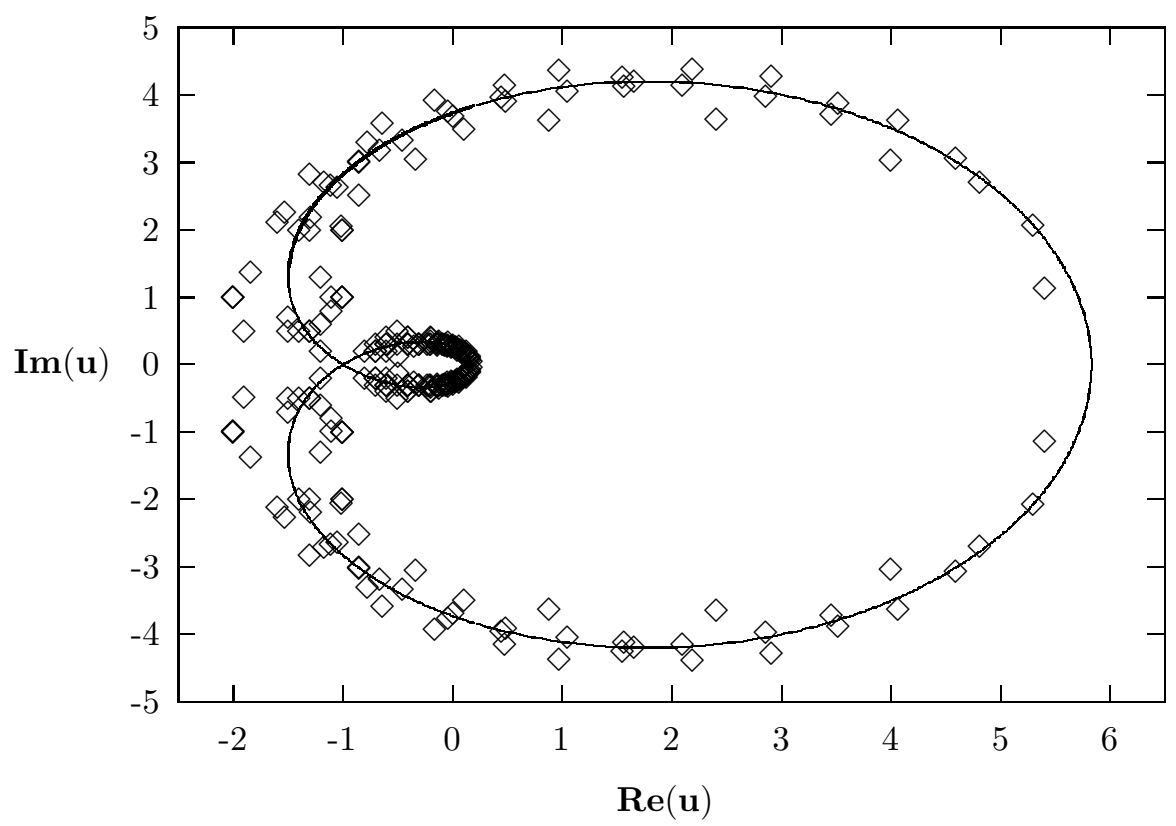

Fig. 2. The complete set of partition function zeros for $L=16$ in the complex $u$ plane (diamonds). The continuous line, parametrized by $\operatorname{Re}(u)=1+2^{3 / 2} \cos w+2 \cos 2 w$ and $\operatorname{Im}(u)=2^{3 / 2} \sin w+$ $2 \sin 2 w$, for $0 \leq w<2 \pi$ corresponds to the phase boundaries (Ref. 17).

The lattice sizes were chosen to explore finite size corrections by an alternative method which will be presented in the next section. In addition, we plot in Fig. 2 all zeros for $L=16$ obtained with MATHEMATICA and compare them with the expected phase boundaries in the $u$ plane for $L \rightarrow \infty .^{17,18}$ This curve corresponds to the locus of points where the free energy is non-analytic and it is parametrized by $\operatorname{Re}(u)=1+2^{3 / 2} \cos w+2 \cos 2 w$ and $\operatorname{Im}(u)=2^{3 / 2} \sin w+2 \sin 2 w$, for $0 \leq w<2 \pi$.

\section{Finite size scaling analysis}

The systematic dependence of $\nu$ on finite systems can be explored to evaluate the main correction to scaling. From pairs of lattices $L$ and $L^{\prime}$, we define the corresponding finite size estimators,

$$
\frac{1}{\nu_{L, L^{\prime}}}=\ln \left(\frac{\left|u_{1}^{0}\left(L^{\prime}\right)-u_{c}\right|}{\left|u_{1}^{0}(L)-u_{c}\right|}\right) / \ln \left(\frac{L}{L^{\prime}}\right) .
$$

This equation was already used to estimate the critical exponent $\nu$ for the 3D Ising model from increasing pairs of lattices. ${ }^{19}$

In Table 2 we present sequences of $1 / \nu_{L, s L}$ as a function of a fixed rescaling factor $s=L^{\prime} / L$. As $\min \left(L, L^{\prime}\right)$ increases, the values obtained by matching pairs of 
Table 2. Sequence of estimates for $1 / \nu_{L, s L}$.

\begin{tabular}{ccccc}
\hline$L / s$ & 1.5 & 2 & 3 & 4 \\
\hline 4 & 1.131948204 & 1.107535391 & 1.083907993 & 1.071996844 \\
6 & 1.067288812 & 1.055806270 & 1.044309560 & 1.038354523 \\
8 & 1.043516688 & 1.036458296 & 1.029248898 & 1.025453193 \\
10 & 1.031641084 & 1.026690577 & 1.021565572 & 1.018835580 \\
12 & 1.024655805 & 1.020902776 & 1.016977082 & 1.014867660 \\
16 & 1.016924350 & 1.014448089 & 1.011818989 & 1.010388613 \\
20 & 1.012804317 & 1.010980582 & 1.009024731 & \\
24 & 1.010266064 & 1.008832544 & & \\
32 & 1.007324514 & 1.006329138 & & \\
40 & 1.005681181 & & & \\
\hline
\end{tabular}

lattices approach the expected limiting value $\nu=1$.

Equation (8), beyond being quite important to estimate $\nu$ can be a starting point to evaluate finite size corrections, which can be due to a variety of sources. ${ }^{20,21}$ For this end let us briefly recall Nightingale's finite size RG transformation.

Under the hypothesis the system is large enough to consider the scaling relation for the longitudinal correlation length $\xi_{L}(\beta)$, the standard expression for the correlation exponent $\nu$ is ${ }^{22,23}$

$$
1+\frac{1}{\nu_{L, L^{\prime}}}=\ln \left(\frac{\partial \xi_{L^{\prime}} / \partial \beta}{\partial \xi_{L} / \partial \beta}\right)_{\beta_{c}} / \ln \left(\frac{L^{\prime}}{L}\right),
$$

where the scaling equation for the correlation length is given by

$$
\xi_{L}=L Y_{\xi}\left(\left(\beta-\beta_{c}\right) L^{1 / \nu}, h L^{y_{H}}, u L^{y_{3}}\right) .
$$

This differentiable equation includes corrections due to the leading bulk irrelevant scaling field $u$ with exponent $y_{3}<0$, and a magnetic field dependence for the sake of completeness.

From Eq. (9) and Eq. (10) one obtains, for $h=0$,

$$
\frac{1}{\nu_{L, L^{\prime}}}=\frac{1}{\nu}+a_{0} \frac{L^{\prime y_{3}}-L^{y_{3}}}{\ln \left(L^{\prime} / L\right)}+b_{0} \frac{L^{\prime 2 y_{3}}-L^{2 y_{3}}}{\ln \left(L^{\prime} / L\right)}+\ldots
$$

where $a_{0}$ and $b_{0}$ include derivatives like $\partial Y_{\xi}(y, z) /\left.\partial y\right|_{y=0, z=0}$. If we replace $L \rightarrow$ $L^{\prime}-1$ in the above equation, one obtains Privman and Fisher's results. ${ }^{23}$

Our analysis follows with the introduction of the rescaling factor $s$ in Eq. (11). The exponent $\nu$ can be asymptotically obtained from sequences either by extrapolating $\ln s \rightarrow \infty$ or by extrapolating $\ln s \rightarrow 0$ (see the similar scaling behaviour of Binder's function ${ }^{24} W_{\beta}^{*}$ for obtaining $2 \beta / \nu$ ). The exponent $y_{3}$ can be evaluated through a linear regression for finite lattice sizes $L$ if we fix the ratio $s$. At this point we call attention to the known fact that the main finite size correction for the 2D Ising model comes from nonlinear scaling fields ${ }^{23,25}$ and gives origin to different $L$-dependent corrections in Eq. (11). 
Table 3. Estimates of $w$ from $1 / \nu_{L, s L}$ data in Table 2. $\tilde{w}$ refers to $1 / \nu_{L, s L}$ data obtained with the replacement $\left|u_{1}^{0}(s L)-u_{c}\right| /\left|u_{1}^{0}(L)-u_{c}\right|$ by $\operatorname{Im} u_{1}^{0}(s L) / \operatorname{Im} u_{1}^{0}(L)$ in Eq. 8 .

\begin{tabular}{cccccc}
\hline$s$ & $L$ & $-w$ & $-\ln a(s)$ & $-\tilde{w}$ & $-\ln \tilde{a}(s)$ \\
\hline 1.5 & $(4,6,8,10,12,16,20,24,32,40)$ & 1.34903 & 0.284605 & 1.09706 & 0.586739 \\
1.5 & $(12,16,20)$ & 1.28388 & 0.514528 & 1.05752 & 0.716982 \\
1.5 & $(12,16,20,24,32,40)$ & 1.21665 & 0.698876 & 1.03807 & 0.770224 \\
1.5 & $(20,24,32,40)$ & 1.17201 & 0.850741 & 1.02581 & 0.811914 \\
1.5 & $(32,40)$ & 1.13858 & 0.970497 & 1.01865 & 0.837543 \\
& & & & & \\
2 & $(4,6,8,10,12,16,20,24,32)$ & 1.35083 & 0.458201 & 1.09585 & 0.735457 \\
2 & $(12,16,20)$ & 1.26133 & 0.735541 & 1.05021 & 0.873225 \\
2 & $(12,16,20,24,32)$ & 1.21780 & 0.853411 & 1.03808 & 0.906048 \\
2 & $(20,24,32)$ & 1.17102 & 1.00516 & 1.02555 & 0.946659 \\
& & & & & \\
3 & $(4,6,8,10,12,16,20)$ & 1.37931 & 0.624774 & 1.10630 & 0.903252 \\
3 & $(12,16,20)$ & 1.23804 & 1.00130 & 1.04348 & 1.06970 \\
& & & & & \\
4 & $(4,6,8,10,12,16)$ & 1.39591 & 0.736397 & 1.11314 & 1.01358 \\
4 & $(12,16)$ & 1.24609 & 1.11215 & 1.04606 & 1.18084 \\
\hline
\end{tabular}

Let us call $w$ the effective exponent coming from the equation

$$
\ln \left(\frac{1}{\nu_{L, s L}}-\frac{1}{\nu}\right)=w \ln L+\ln a(s),
$$

which intends to detect the main correction regardless its origin.

We collect in Table 3 our results for $w$. The third and fourth columns correspond to fit Eq. (12) to data of Table 2.

For the $2 \mathrm{D}$ Ising model $u_{c}$ is exactly known, however for many models the value of $u_{c}$ is not known with high precision and in this case it is usual to replace $\left|u_{1}^{0}-u_{c}\right|$ by $\operatorname{Im} u_{1}^{0}$. For sake of illustrative purposes both cases were considered. In our tables we use the notation $\tilde{w}$ instead of $w$ when the $1 / \nu_{L, s L}$ data is obtained from Eq. (8) with the replacement $\left|u_{1}^{0}(s L)-u_{c}\right| /\left|u_{1}^{0}(L)-u_{c}\right|$ by $\operatorname{Im} u_{1}^{0}(s L) / \operatorname{Im} u_{1}^{0}(L)$. Different fixed ratios $s$ are used to show the behaviour of $w$. As $s$ increases, and consequently $L^{\prime}$, the numerical results show a trend in direction of $w=-1$. Large $s$ means working with crossings involving a large $L$, hence close to $\beta_{c}$. The corresponding best linear fits for all data are presented in Fig. 3. It is clear that small lattice sizes give origin to deviations in the employed linear equation (12).

To complete our analysis we present in Table 4 the dependence of $w$ on small lattices. The value $w \approx-1.7$ is quite close to Binder's reported value, $w \approx-1.8$, as the main correction to the function $W_{\beta}^{*} \cdot{ }^{24,26}$ However there smaller lattices were considered in the analysis which seem to increase $|w|$. This trend can be caught from our smaller data set with $s=1.5$ for $L=4$ and 6 .

In summary, we have described an approach to compute partition function zeros for large lattice sizes. Although it was applied to the exact 2D Ising model partition function, it can be quite useful either when we deal with large coefficients, even out of scope of double precision computations, or when the polynomial has a large number 


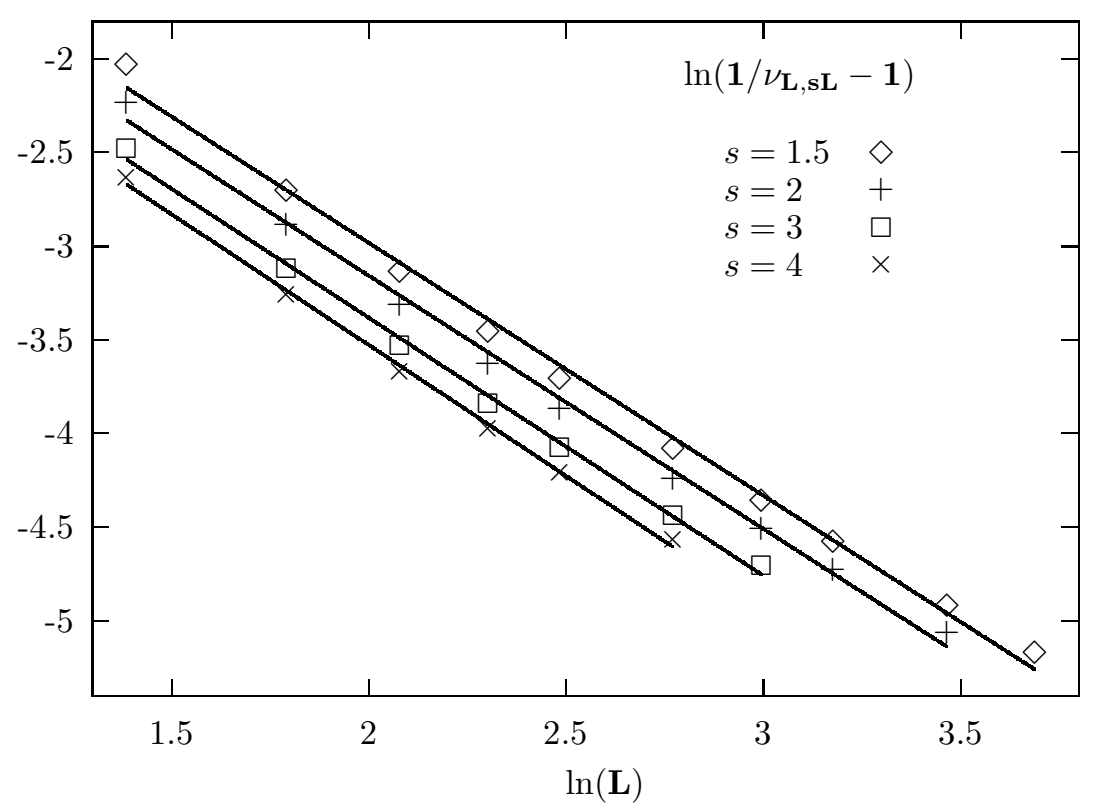

Fig. 3. Linear regressions for $\ln \left(1 / \nu_{L, s L}-1\right)$ for several values of the rescaling factor $s$, according to Eq. (12).

of coefficients, which would prevent us from using standard solving algorithms. This last situation is proper for the multicanonical simulation of the density of states in the 3D Ising model. ${ }^{27}$

In MC simulations FSS behaviour of the first zero has been used to estimate the exponent $\nu$ and the critical coupling $\beta_{c}$. Beyond any MC data, with limited statistical precision, we were able to explore here the performance of the FSS analysis proposed to evaluate corrections to scaling. This approach reveals to be quite

Table 4. Estimates of $w$ as in Table 3.

\begin{tabular}{cccccc}
\hline$s$ & $L$ & $-w$ & $-\ln a(s)$ & $-\tilde{w}$ & $-\ln \tilde{a}(s)$ \\
\hline 1.5 & $(4,6)$ & 1.66085 & -0.277077 & 1.27701 & 0.268877 \\
1.5 & $(4,6,8)$ & 1.60430 & -0.191966 & 1.23731 & 0.328639 \\
1.5 & $(4,6,8,10)$ & 1.56144 & -0.123144 & 1.20877 & 0.374454 \\
& & & & & \\
2 & $(4,6)$ & 1.61773 & $-1.27172 \times 10^{-2}$ & 1.24410 & 0.478115 \\
2 & $(4,6,8)$ & 1.56425 & $6.77855 \times 10^{-2}$ & 1.20847 & 0.531757 \\
2 & $(4,6,8,10)$ & 1.52369 & 0.132909 & 1.18311 & 0.572470 \\
\hline
\end{tabular}

useful in setting an upper limit on $w$ for the same data set as $s$ increases, as can be observed, for example, from our Table 3 for $L=12,16$ and 20 . 


\section{Acknowledgements}

Nelson Alves and Drugowich de Felicio are supported by the Brazilian agencies CNPq and FAPESP. Numerical calculations were performed in part on the AlphaAXP 3000/300X station at FFCLRP and in part on the Alpha-AXP 3000/800 at Departamento de Física Matemática (IFUSP) in São Paulo.

\section{References}

1. P. D. Beale Phys. Rev. Lett. 76 (1996) 78.

2. B. Kaufman Phys. Rev. 76 (1949) 1232.

3. C. Itzykson, R. B. Pearson and J. B. Zuber Nucl. Phys. B 220 [FS8] (1983) 415.

4. S. Katsura Prog. Theor. Phys. 38 (1967) 1415.

5. Y. Abe and S. Katsura Prog. Theor. Phys. 43 (1970) 1402.

6. M. E. Fisher, Lectures in Theoretical Physics, vol. 7C p. 1 (University of Colorado Press, Boulder, 1965).

7. M. Suzuki, C. Kawabata, S. Ono, Y. Karaki and H. Ikeda J. Phys. Soc. Japan 29 (1970) 837.

8. S. Katsura, Y. Abe and M. Yamamoto J. Phys. Soc. Japan 30 (1971) 347.

9. G. Bhanot J. Stat. Phys. 60 (1990) 55; R. J. Creswick, Phys. Rev. E 52 (1995) R5735.

10. M. Falcioni, E. Marinari, M. L. Paciello, G. Parisi, B. Taglienti Phys. Lett. B 108 (1982) 331.

11. K. M. Bitar Nucl. Phys. B 300 [FS22] (1988) 61.

12. N. A. Alves, B. A. Berg and S. Sanielevici Nucl. Phys. B 376 (1992) 218.

13. E. Marinari Nucl. Phys. B 235 [FS11] (1984) 123.

14. G. Bhanot, R. Salvador, S. Black, P. Carter and R. Toral Phys. Rev. Lett. 59 (1987) 803.

15. N. A. Alves, B. A. Berg and R. Villanova Phys. Rev. B 41 (1990) 383.

16. W. H. Press et al., Numerical Recipes: The Art of Scientific Computing, (Cambridge Univ. Press, London, 1989).

17. V. Matveev and R. Shrock J. Phys. A: Math. Gen. 28 (1995) 1557.

18. V. Matveev and R. Shrock Phys. Rev. E 53 (1996) 254.

19. N. A. Alves, B. A. Berg and R. Villanova Phys. Rev. B 43 (1991) 5846.

20. F. J. Wegner Phys. Rev. B 5 (1972) 4529.

21. A. Aharony and M. E. Fisher Phys. Rev. B 27 (1983) 4394.

22. E. Brèzin J. Physique 43 (1982) 15.

23. V. Privman and M. E. Fisher J. Phys. A: Math. Gen. 16 (1983) L295.

24. K. Binder Z. Phys. B 43 (1981) 119.

25. A. Aharony and M. E. Fisher Phys. Rev. Lett. 45 (1980) 679.

26. K. Binder Phys. Rev. Lett. 47 (1981) 693.

27. N. A. Alves, J. R. Drugowich de Felicio and U. H. E. Hansmann (unpublished). 